Stoa

Vol. 3, no. 5, 2012, pp. 195-221

ISSN 2007-1868

\title{
LA METATEORÍA ESTRUCTURAL Y LA LINGÜÍSTICA: EL INICIO DEL CASO DE ZELLIG HARRIS
}

Luis Miguel Peris-Viñé

Facultad de Filosofía y Letras

Universidad de Granada

perisv@ugr.es

RESUMEN: Analizamos aspectos centrales de la gramática distribucional de Zellig Harris, (la estructura distribucional, los procedimientos de determinación de los elementos fonológicos y morfológicos y el análisis transformacional) para preparar el camino a su reconstrucción futura mediante la metateoría estructural. Propondremos un modo de concebir el dominio de la teoría, identificaremos sus nociones básicas, los procedimientos mediante los que se elaboran los conjuntos auxiliares y propondremos la forma general de su ley fundamental.

PALABRAS CLAVE: Harris · Chomsky · lingüística estructural · reconstrucción estructural $\cdot$ análisis transformacional

ABSTRACT: We analyze central aspects of the distributional grammar of Zellig Harris (the distributional structure, the procedures of determination of the phonological and morphological elements and the transformational analysis) to prepare its future reconstruction by means of the structural metatheory. We will propose a way to understand the domain of the theory, we will identify its basics notions, the methods used to elaborate the auxiliary sets and we will also propose the general form of its fundamental law.

KEYWORDS: Harris · Chomsky · structural linguistic $\cdot$ structural reconstruction - transformational analysis

\section{Presentación}

El objetivo de este trabajo es ilustrar algunas de las posibilidades que la metateoría estructural ofrece para la reconstrucción de las teorías lingüísticas. Abordaremos este objetivo analizando ciertos aspectos de la gramática distribucional de Zellig Harris (GD) y su relación con la gramática generativa transformacional de Noam Chomsky (GCH) tal y como estas teorías se desarrollaron en la década de los años cincuenta del 
siglo xx. Estamos convencidos de que las teorías lingüísticas más influyentes del siglo xx se dejan analizar mediante la metateoría estructural y de que además ese análisis resulta ser relevante para abordar y resolver cuestiones fundamentales sobre la naturaleza de esas teorías, cuestiones relativas, por ejemplo, a las relaciones que tales teorías mantienen entre sí y que revelan aspectos de su fundamentación. ${ }^{1}$

Las relaciones entre la corriente estructuralista en lingüística y la corriente generativa transformacional (y en especial entre la gramática de Harris y la gramática de Chomsky) no han sido objeto de suficiente atención en filosofía de la lengua, pese a que su consideración constituye una piedra de toque para cualquier acercamiento metateórico a la lingüística como disciplina. La naturaleza de estas dos teorías puede apreciarse si captamos cuál fue la verdadera relación que se dio entre ellas, lo cual arrojará luz sobre la naturaleza de la lingüística en su conjunto. La manera usual de concebir esta relación la presenta más en términos de diferencias e incluso de oposiciones que en términos de semejanzas. Por ejemplo: empirismo frente a tolerancia epistemológica y prioridad teórica (en cuanto a la metodología), nominalismo frente a conceptualismo (en cuanto a la concepción del lenguaje), informes de estado frente a teorías (en cuanto a los resultados). Nuestro punto de vista, defendido en este trabajo y en otros, es que la relación entre GD y GCH se produjo en términos de semejanzas y de influencias. ${ }^{2}$ Por ello, nuestras consideraciones sobre la relación entre ambas teorías se enmarcan en un objetivo más general: contribuir a defender que en el paso de Harris a Chomsky no se produce una revolución sino algún proceso de cambio de teorías conmensurables.

En nuestro análisis de GD atenderemos a especialmente a la estructura distribucional propuesta por Harris, a los procedimientos de determinación de los elementos fonológicos y morfológicos, y a su análisis transformacional. No ofreceremos una reconstrucción en sentido estricto, pormenorizada y compacta, de los diversos aspectos considerados, sino que indicaremos y analizaremos en detalle aquellos aspectos de GD que una reconstrucción estructural deberá abordar y argumentaremos sobre el modo en que esa reconstrucción deberá proceder. En especial, nos detendremos en la configuración de lo que sería

${ }^{1}$ Cfr. Peris-Viñé 1996, 2011 y 2012b.

${ }^{2}$ Cfr. Peris-Viñé 1997, 2002 y 2003. 
el conjunto base principal de GD, los conjuntos auxiliares sobre los que definir sus nociones básicas, la nociones básicas resultantes y la forma general de su ley fundamental. Una consideración detenida merecerá el análisis transformacional de Harris, pues su adecuada comprensión e inserción en los desarrollos de la lingüística del siglo xx es una condición para la correcta reconstrucción tanto de GD como de GCH.

\section{Método y teoría}

En Harris (1951), ${ }^{3}$ el mejor exponente de la metodología propia de la lingüística estructuralista, se señala que su interés se dirige a las operaciones y procedimientos de análisis más que a los resultados de ese análisis. Esto, que es una característica de Harris (1951), es también, en cierta medida, uno de los aspectos constitutivos de la corriente estructuralista en lingüística. El estructuralismo en lingüística es ante todo un método. El propósito no es tanto lograr una teoría precisa como usar un método preciso, de ahí que haya un especial interés en caracterizar antes que nada dicho método. Este rasgo de la lingüística estructural brinda a la filosofía de la lingüística un excelente campo para los análisis metodológicos y de fundamentación, y, por contra, hace más ardua la tarea de identificar estructuras teóricas. Así pues, los componentes teóricos que se encuentran en las propuestas de Harris están a veces expresados en contornos no precisos o sólo implícitamente. Ello hará que la tarea de reconstruirlos, de tener éxito, adquiera un valor añadido.

El llamado método de GD consiste en procedimientos de análisis distribucional. En el caso de GCH encontramos algo con un papel seme-

\footnotetext{
${ }^{3}$ Ya desde su misma publicación esta obra fue considerada como un hito en la evolución de la lingüística, en tanto que culmen de su pasado reciente y en tanto que guía de las investigaciones que le siguieron ( $c f r$. McMquown 1952, Hymes y Fought 1981, Plötz 1972 y Katz 1981). Los rasgos característicos de la concepción filosófica de Harris pueden rastrearse considerando sus ideas sobre: lo que es el lenguaje y lo que es una gramática; el procedimiento de validación de juicios de los hablantes; el carácter no científico de la lingüística; la naturaleza de las transformaciones; el análisis del discurso; su instrumentalismo. El trasfondo ontológico nominalista del estructuralismo se manifiesta tanto en Leonard Bloomfield como en Harris, pero en éste los compromisos empiristas son menos fuertes que en aquél. Así, aunque para Harris (1951, pp. 4-5) los datos de la lingüística proceden de la observación de la conducta lingüística no por ello se compromete, como hace Bloomfield, con el criterio empirista de significado. Harris no adopta un compromiso con la verdad y acepta que análisis alternativos puedan ser válidos.
} 
jante al método de Harris. Se trata de los procedimientos generativos. En atención a cómo se usan en ambas teorías, la aplicación de estos procedimientos da lugar, en cada caso, a tres tipos de logros teóricos sobre los que una reconstrucción futura desde la metateoría estructural tendrá que operar: la elaboración de los componentes empleados en la descripción (los conjuntos base auxiliares), la descripción de porciones del lenguaje mediante esos componentes (la asignación a los objetos del dominio de los valores correspondientes a las nociones básicas) y la caracterización de un lenguaje en su totalidad (una descripción compacta, que atienda a los rasgos propios de un lenguaje y que se exprese en una ley fundamental).

En Harris y en Chomsky estos tres tipos de logros se desarrollan con diferente complejidad y alcance. En este trabajo analizaremos los procedimientos y los logros correspondientes a GD y asumiremos el análisis de los procedimientos y logros correspondientes a GCH recogido en Peris-Viñé (2011). Pero antes veremos que, pese a su papel semejante, los procedimientos de análisis distribucional y los procedimientos generativos son de naturaleza muy distinta y ofrecen la clave para entender las diferencias más sustantivas pero también las vinculaciones más significativas entre ambas teorías.

\section{Procedimientos de análisis y procedimiento generativos: relación entre GD y GCH}

En GD el punto de partida lo constituyen las proferencias de un lenguaje y el interés se dirige a desarrollar procedimientos de análisis que permitan determinar la estructura de esas proferencias respecto de diferentes niveles de representación; por contra, en GCH el interés se dirige a desarrollar procedimientos generativos que permitan derivar las proferencias y expresar su estructura. En un caso el resultado es una gramática concebida como un sistema de representación y en el otro el resultado es una gramática concebida como un sistema de generación. Esta diferencia, teniendo en cuenta la perspectiva desde la que se estudia la estructura de las proferencias, se puede caracterizar diciendo que los modelos matemáticos ejemplificados en cada una son distintos: en GD se hace uso de modelos analíticos mientras que en GGH se utilizan modelos sintéticos. Marcus (1967, p. vii) da cuenta de esta diferencia, y señala que: 
There are two fundamental types of models which are studied in algebraic linguistics: generative and analytic. Simplifying, we might say that within the framework of a generative model, the starting point is a certain grammar, while the object we study is the language generated by this grammar. An analytic model presents an inverse situation; here the starting point is a certain language, i.e., a certain collection of sentences, whereas the purpose of the study is to establish the structure of these sentences, their constitutive elements, and the relations among them within the framework of sentences. ${ }^{4}$

En nuestra opinión, el propio Harris se hace eco de esta diferencia al hablar de to state regularities y de to synthesize utterances en Harris (1951, p. 365, 372) y de item style y process style en Harris (1954, p. 6). Para él se trata de simplemente de "a choice of purposes facing the investigator in linguistics" (Harris 1951, p. 365) Incluso podría decirse que para Harris los modelos sintéticos presuponen los modelos analíticos. Así nos dice:

The work of analysis leads right up to the statements which enable anyone to synthesize or predict utterances in the language. These statements form a deductive system with axiomatically defined initial elements and with theorems concerning the relations among them. The final theorems would indicate the structure of the utterances of the language in terms of the preceding parts of the system. (Harris 1951, pp. 372-373)

En esta línea de conexión entre ambos sistemas, el propio Chomsky (1955) va más lejos incluso y establece una dependencia entre sistemas de representación y sistemas de generación al formular su propuesta. Para Chomsky (1955) una gramática generativa (formulada como un conjunto de reglas) debe expresar la información sobre las proferencias que proporciona la correspondiente gramática analítica (formulada como un sistema algebraico de niveles de representación). "For each linguistic level, we show how the information about utterances provided on this level can be presented as a sequence of conversions, and how the underlying algebra (i.e., the structure of the level) can be reconstructed from the sequence of conversions" (Chomsky 1955, pp. 67-68). "Then, given a corpus, we can construct a set of compatible levels, each with the proper internal structure, and such that the

${ }^{4}$ Cfr. también Serrano 1975. 
correlated sequence of conversions produces the corpus (along with much else)" (Chomsky 1955, p. 68). Es decir, dada una proferencia y la representación que una gramática analítica le asigna, desde la correspondiente gramática generativa se debe poder construir, aplicando las correspondientes conversiones o reglas generativas, una derivación de dicha proferencia, derivación que además exprese la información contenida en la representación que la gramática analítica asignó a la proferencia.

Si partimos de que una gramática analítica, como GD, se articula en un sistema de niveles de representación que ofrece diferentes representaciones para una misma proferencia, podemos entender la propuesta de Chomsky cuando nos dice que "We must be able to recover from a grammar a sequence of representations $\left(R_{1}, \ldots, R_{n}\right)$ for each sentence, where $R_{1}$ is the representation Sentence, $R_{n}$ is a phonetic spelling, and $R_{2}, \ldots, R_{n-1}$ are intermediate representations in terms of phrases, words, phoneme, etc. We can generate these representation sequences by rules of the form (...) X $\rightarrow Y$ interpreted as the instruction "rewrite $X$ as $Y$ ". We call each such rule a conversion. The string $Z^{\wedge} Y^{\wedge} W$ is said to follow from the string $Z^{\wedge} X^{\wedge} W$ (where $Z, W$, or both may be the identity element $U$ ) by the conversion $[X \rightarrow Y](\ldots)$. We say that the sequence $\left(R_{1}, \ldots, R_{n}\right)$ is a derivation of $R_{n}$, generated by a set $C$ of conversions, if $R_{1}$ is Sentence and for each i $(1 \leq i<n), R_{i+1}$ follows from $R_{i}$ by one of the conversions of $C$ " (Chomsky 1955, p. 114). Así pues, para cada nivel de representación de una gramática analítica habrá que especificar el conjunto de reglas de la gramática generativa correspondiente que permite generar la representación asignada desde ese nivel. El modo en el que Chomsky (1955) se expresa en estos y otros pasajes puede interpretarse como una manera de defender que una gramática generativa, concebida como un sistema de reglas, puede proporcionar cuando menos el análisis que venía siendo realizado desde la gramática distribucional de Harris.

Estas consideraciones nos sirven también para apreciar la inexactitud de la usual afirmación de que una gramática generativa consiste, sin más, en un conjunto organizado o sistema de niveles de representación, pues, en sentido estricto, en Chomsky (1955) son procedimientos diferentes, aunque el resultado de su aplicación se pretende sea equivalente como condición de validez de la gramática generativa en cuestión. 
Esta correspondencia entre gramáticas analíticas y gramáticas generativas le permite a Chomsky (1955) estudiar ciertos aspectos de los sistemas de representación a partir de las gramáticas generativas correspondientes. En especial le permite detectar algunas de las deficiencias de los sistemas de representación de la tradición estructural, en concreto las deficiencias cuya solución aconseja introducir un nuevo nivel de representación, el transformacional; es decir, el tipo de gramática (que depende del tipo de reglas que incorpora) manejado hasta la introducción de las reglas transformacionales se mostrará inadecuado una vez comprobadas las ventajas de introducir en la gramática tales reglas transformacionales. Esto llevará a Chomsky (1955) a caracterizar el nivel de representación correspondiente a este nuevo tipo de reglas, el nivel de representación transformacional.

Esta conexión entre la gramática generativa de Chomsky y la gramática analítica de Harris muestra en realidad una importante vía de dependencia entre ambas y es clave para apreciar sus interrelaciones. Así, podría decirse que mientras el punto de partida de la gramática de Harris es un lenguaje, el punto de partida de la gramática de Chomsky es doble: un lenguaje y la gramática analítica de Harris correspondiente. Vista desde otra perspectiva, esta dependencia propuesta por Chomsky habría que formularla diciendo que los procedimientos distribucionales de GD son procedimientos de determinación mientras que los procedimientos generativos de $\mathrm{GCH}$, las reglas de una gramática generativa, son más bien meros procedimientos de asignación cuyos resultados deben coincidir con los valores determinados por los procedimientos distribucionales de la correspondiente y previa gramática analítica.

Expresada así, esta perspectiva de la dependencia entre GD y GCH que como hemos visto el propio Chomsky (1955) postula, con ser interesante, podría identificarse de modo más ajustado y certero si hacemos uso de los recursos de la metateoría estructural, en concreto de la distinción entre nociones $T$-teóricas y $T$-no-teóricas. Aunque no es posible detenernos en ello, hay razones para defender que ciertas nociones de GCH aparecen ya en GD y son GCH-no-teóricas mientras que otras son propias de GCH y funcionan como GCH-teóricas. Por tanto, la dependencia entre estas dos teorías es más compleja de lo que postula Chomsky (1955), pues mientras que respecto de las nociones GCH-noteóricas podríamos decir que $\mathrm{GCH}$ pretende formular reglas que repro- 
duzcan y asignen sus valores, previamente determinados quizás en GD o en alguna otra teoría anterior, en cambio, respecto de las nociones GCH-teóricas los procedimientos de determinación sí serían las propias reglas de $\mathrm{GCH}$.

De cualquier forma, por los textos que acabamos de citar, independientemente de los procedimientos de obtención a emplear, se evidencia que Harris considera la tarea de derivar proferencias como lícita e integrable en su concepción de la lingüística y, por otra parte, que Chomsky (1955) considera que sus propias propuestas están en la línea de las propuestas previas de Harris. Por tanto, la derivación de proferencias, clave en $\mathrm{GCH}$, no es un aspecto que oponga o enfrente radicalmente esa teoría con la corriente estructuralista en lingüística y en particular con GD. Más adelante, cuando consideremos los procedimientos concretos de GD y los logros a los que conducen será el momento de indicar cómo puede la metateoría estructural dar cuenta de ellos. Pero antes debemos precisar cuál es el dominio de la teoría.

\section{Universo del discurso y datos de la lingüística: conjunto base principal}

La identificación del dominio de la teoría requiere tener claro la procedencia y la naturaleza de los datos, lo que en el caso de GD exige considerar y analizar la importante noción de corpus. Los datos de la lingüística estructural proceden de la conducta lingüistica. En la conducta lingüística se producen una serie de movimientos en los órganos articulatorios del hablante, lo que da lugar a ondas sonoras que se convierten en impresiones acústicas para el oyente. El universo del discurso para GD es un lenguaje o dialecto particular, el lenguaje nativo de una comunidad de habla. Y se estudia sin considerar los cambios que en él se producen a lo largo del tiempo, es decir, se estudian los lenguajes desde la perspectiva sincrónica. ${ }^{5}$ GD estudia el lenguaje atendiendo a proferencias completas de ese lenguaje. Es decir, el universo del discurso está integrado por proferencias completas.

\footnotetext{
${ }^{5}$ Aparte de la perspectiva diacrónica, otros aspectos que también son consustanciales a una lengua y de los que también hace abstracción el estructuralismo son la variación dialectal y las diferencias de estilo. Estas desviaciones de la norma pueden ser asumidas mediante una variación en el sistema o mediante un sistema distinto e independiente del sistema propuesto para el dialecto mayoritario, es decir, al estructuralismo no le interesa descubrir y estudiar tales desviaciones de la norma.
} 
An utterance is any stretch of talk, by one person, before and after which there is silence on the part of the person. The utterance is, in general, not identical with the sentence' (as that word is commonly used), since a great many utterances, in English for example, consist of single words, phrases, 'incomplete sentences', etc. Many utterances are composed of parts which are linguistically equivalent to whole utterances occurring elsewhere (Harris 1951, p. 14).

Por tanto, no se consideran situaciones reales de habla, discursos, en donde distintas proferencias pueden sucederse por la acción de varios interlocutores.

Normalmente, el lingüista no estudia proferencias aisladas, sino en conjunto, un corpus:

Investigation in descriptive linguistics consists of recording utterances in a single dialect and analyzing the recorded material. The stock of recorded utterances constitutes the corpus of data, and the analysis which is made of it is a compact description of the distribution of elements within it. The corpus does not, of course, have to be closed before analysis begins. [...] To persons interested in linguistic results, the analysis of a particular corpus becomes of interest only if it is virtually identical with the analysis which would be obtained in like manner from any other sufficiently large corpus of material taken in the same dialect. If it is, we can predict the relations among elements in any other corpus of the language on the basis of the relations found in our analyzed corpus. When this is the case, the analyzed corpus can be regarded as a descriptive sample of the language. How large or variegated a corpus must be in order to qualify as a sample of the language, is a statistical problem; it depends on the language and on the relations which are being investigated (Harris 1951, pp. 12-13).

En relación a lo dicho, merece que hagamos alguna consideración en torno a las nociones de corpus y de proferencia para apreciar algunas diferencias y semejanzas entre GD y GCH. El pasaje recién citado de Harris parece oponerse a algunas de las ideas tópicas difundidas como parte de la historia oficial sobre el estructuralismo en lingüística. Según esta visión tópica, las gramáticas de los estructuralistas estarían confinadas a serlo sólo de un determinado corpus de partida (no de la totalidad de la lengua), mientras que las gramáticas generativas transformacionales, no partirían de un corpus, que por definición es limitado, sino que se ocuparían de toda una lengua y formularían 
la competencia del hablante sobre una infinidad de oraciones. Sin embargo a partir de este pasaje queda claro para Harris que el corpus no se considera cerrado, que hay una clara diferencia entre un corpus y la totalidad de la lengua, que hay corpus más informativos que otros y que el más informativo de estos [sample], el que permite apreciar las relaciones de toda una lengua, sirve para predecir sobre la totalidad de la lengua. Por otro lado, además, el propio Chomsky defiende que la tarea del lingüista ha de partir de la consideración de un corpus finito desde el que elaborar una gramática válida para todo el lenguaje en el que se incluye ese corpus. "Then, given a corpus, we can construct a set of compatible levels, each with the proper internal structure, and such that the correlated sequence of conversions produces the corpus (along with much else)" (Chomsky 1955, p. 68). Según Chomsky (1955, p. 129)

The first problem that the linguist must face in constructing the grammar of a language is that of determining the subject matter of his description. Given a corpus of sentences, this problem breaks down into two parts [...] First, he must determine which of these utterances are phonemically distinct. Second, he must determine which utterances, whether in the corpus or not, are grammatical. ${ }^{6}$

Por lo dicho sobre la noción de corpus podemos concluir que tanto en GD como en GCH el corpus es el punto de partida desde el cual elaborar la gramática.

Visto desde la filosofía de la ciencia, el corpus del que parte el lingüista ha de ser concebido de dos maneras: como un campo de cultivo desde el cual desarrollar las nociones con las que después se describa el lenguaje y como un campo de contrastación; es una cosa distinta en cada una de estas formas de concebirlo y cumple una función distinta. El corpus de cultivo es una porción de habla, más o menos fragmentaria, seleccionada mediante criterios no necesariamente sistemáticos por el lingüista con la intención de obtener los conjuntos base principales de la teoría. Por su parte, el corpus de contrastación es un conjunto de posibles aplicaciones de la teoría de las que el lingüista pretende dar cuenta con la intención de establecer y ampliar el alcance de la teoría. Es bien sabido que al corpus, en tanto que campo de cultivo, Harris le

${ }^{6}$ Cfr. también Chomsky 1955, pp. 61, 78, 147, 157, entre otros lugares. 
aplica procedimientos distribucionales de análisis. Lo que no es sabido, o tomado en cuenta, es que Chomsky (1955), al proponer que sus procedimientos generativos reproduzcan el análisis efectuado desde una gramática distribucional, asume los resultados de este análisis distribucional, pues necesita disponer previamente de los conjuntos base principales sobre los que aplicar sus procedimientos generativos. En cuanto al corpus de contrastación cabe decir que es apreciable tanto en Harris (1951) como en Chomsky (1955), y está integrado por diferentes clases de aplicaciones de la gramática, básicamente oraciones aisladas, oraciones de un cierto tipo y grupos de oraciones parcialmente semejantes. No hay diferencia en este punto entre las gramáticas de Harris y de Chomsky.

\section{Procedimientos concretos de la gramática de Harris}

Los procedimientos de análisis de la gramática de Harris se aplican, en una determinada secuencia, a las proferencias con dos objetivos: identificar sus partes y mostrarlas como ejemplificaciones de unas pocas combinaciones de algunos elementos; consisten en elaboradas técnicas de sustitución de segmentos lingüísticos en atención a su distribución. La aplicabilidad de este método

is based on two suppositions: that the investigator is able to perform an initial segmentation of the speech continuum [...] and that he is able, by substituting his initial segments one for another and by observing a native speaker's reaction, to judge which segments are equivalent for that speaker and which are not. (Mcquown 1952, p. 495)

La distribución "of an element is the total of all environments in which it occurs, i.e. the sum of all the (different) positions (or occurrences) of an element relative to the occurrence of other elements" (Harris 1951, pp. 15-16). El entorno "or position of an element consists of the neighborhood, within an utterance, of elements which have been set up on the basis of the same fundamental procedures which were used in setting up the element in question" (Harris 1951, p. 15). En otras palabras: "An environment of an element $A$ is an existing array of its cooccurrents, i.e., the others elements, each in a particular position, with which $A$ occurs to yield an utterance" (Harris 1954, p. 3). 
Pero Harris, además de interesarse primordialmente por las relaciones de distribución de los elementos del lenguaje, considera que el lenguaje en su totalidad tiene una estructura distribucional (cfr. Harris 1954). Eso significa que el lenguaje puede ser descrito mediante un sistema de enunciados que exprese la totalidad de las relaciones de distribución de sus componentes. Este sistema de enunciados, que constituirá una red de enunciados en la que algunos de ellos se formularán en términos de otros, se organiza en los llamados niveles de descripción. Una reconstrucción de GD deberá caracterizar las entidades descriptoras adecuadas de los distintos niveles, para lo cual tendrá que establecer y definir los conjuntos base auxiliares correspondientes a las distintas nociones relacionales básicas.

Los procedimientos de análisis distribucional de la gramática de Harris se agrupan en dos pasos: los que conducen a la determinación de los elementos relevantes de partida y los que conducen a la identificación de las relaciones de distribución que se dan entre esos elementos. ${ }^{7} \mathrm{La}$ determinación de los elementos debe afrontar el hecho de que "that speech is a set of complex continuous events - talking does not consist of separate sounds enunciated in succession - and the ability to set up discrete elements lies at the base of the present development of descriptive linguistics" (Harris 1951, p. 20). Esos elementos podrán determinarse si somos capaces de delimitar aquellas porciones de las proferencias que son similares en varias de ellas. “'Similar' here means not physically identical but substitutable without obtaining a change in response from native speakers who hear the utterance before and after the substitution: e.g. the last part of He's in. is substitutable for the last part of That's my pin" (Harris 1951, p. 20). Por su parte, las relaciones de distribución pueden identificarse en la medida que apreciemos que la ocurrencia de los elementos se produce en distintos grados de dependencia, es decir, que la frecuencia de los elementos está condicionada, en distinto grado, a ciertos entornos. Y ello permite definir clases de elementos con unas dependencias semejantes. ${ }^{8}$ En una reconstrucción de GD estas clases serán las extensiones de las nociones que se emplearán

\footnotetext{
${ }^{7}$ Nótese que la distribución es un dato, un método y un resultado, es decir, comenzamos apreciando ciertas relaciones de distribución de un supuesto elemento (dato), lo que permite manipular mediante procedimientos distribucionales el flujo del habla (método) a fin de determinar los elementos definitivos y sus relaciones de distribución (resultado).

${ }^{8}$ Cfr. Harris 1954, pp. 16-17.
} 
en la descripción de las proferencias, o si se quiere, las entidades descriptoras; es decir, la identificación de las relaciones de distribución permitirá, finalmente, constituir los conjuntos base auxiliares, a los que pertenecen los valores de las nociones relacionales de GD.

\section{Niveles de descripción: conjuntos base auxiliares y procedimientos de determinación mediante segmentación y distinción}

Las entidades descriptoras de la gramática de Harris pertenecen básicamente a dos niveles de descripción: el fonológico y el morfológico. ${ }^{9}$ La determinación de los elementos en ambos niveles debe dar lugar a dos conjuntos de elementos capaces de representar todas las proferencias: "very utterance can be completely identified as a complex phonemic elements, and every utterance can be completely identified as a complex of morphemic elements" (Harris 1951, p. 21). Aunque varíen los resultados, los métodos utilizados en fonología y morfología son los mismos: asociar elementos discretos con rasgos particulares de porciones de eventos continuos y establecer interrelaciones entre esos elementos.

El nivel desde el que el lingüista obtiene sus datos de partida es el fonético. Una descripción fonética de una proferencia ofrece una descripción física de la misma, independientemente de si las proferencias son consideradas eventos fisiológicos o acústicos. Esa descripción acota porciones del continuo del habla en atención a su articulación y a su sonido, y tales porciones o elementos fonéticos son utilizados por una gramática descriptiva para determinar los elementos propiamente lingüísticos, estos son los fonológicos y los morfológicos. ${ }^{10}$

Para la determinación de los elementos fonológicos se emplean los procedimientos de segmentación y de distinción, los cuales hacen posible disponer de una serie de elementos discretos (distinciones fonémicas, más que fonemas) que se combinan en las proferencias de una lengua.

\footnotetext{
${ }^{9} \mathrm{Si}$ entramos en detalle, hay que decir que para Harris no hay solo dos sistemas descriptivos, la fonología y la morfología, sino un número indefinido de sistemas fonológicos y sistemas morfológicos ( $c f r$. Harris 1951, pp. 364-365). Consecuentemente, no sólo hay fonemas y morfemas, sino también otros elementos como segmentos fonológicos (pertenecientes a un nivel inferior al fonológico) o morfonemas (pertenecientes a un nivel entre el fonológico y el morfológico) o clases de morfemas (pertenecientes a un nivel superior al morfológico). ${ }^{10} \mathrm{Cfr}$. Harris 1951, pp. 4-5.
} 
Mediante el procedimiento de la segmentación se logra representar "the continuous flow of a unique occurrence of speech as a sucession of segmental elements, each representing some feature of a unique speech sound" (Harris 1951, p. 25). Es decir, el propósito es representar una realidad continua mediante una composición de elementos discretos:

Utterances are stretches of continuous events. If we trace them as physiological events, we find various parts of the body moving in some degree independently of each other and continuously [...] If we trace utterances as acoustic events, we find continuous changes of sound-wave periodicities $[\ldots]$ Each of these segments may be described very roughly as the sum of particular coincident movements of speech organs (lip closing, etc.), or as so many sound-wave crests of such and such form (Harris 1951, pp. 25-26).

Una vez determinados los segmentos, le asignamos a cada uno una marca para poder nombrarlos: por ejemplo $k^{h}$ para el primer elemento de Cant't do it. "Each sign corresponds to a unique and particular segment in a particular stretch of speech. And each sign (or the segment which it indicates) is now considered a single element" (Harris 1951, p. 27). Por su parte, el procedimiento de la distinción se dirige a establecer equivalencias lingüísticas, es decir, establecer cuándo cualesquiera dos segmentos son descriptivamente equivalentes. Cuando esto último es el caso se dice que los dos segmentos en cuestión son variantes libres o mutuamente sustituibles. ${ }^{11}$ Una vez establecida una clase de segmentos que funcionan como variantes libres se puede utilizar un único símbolo para referirse a cualquier miembro de la clase, a la vez que pasar por alto aquellas diferencias que hayan podido ser apreciadas previamente entre los segmentos equivalentes de dicha clase. Como es lógico pensar, estos procedimientos de análisis (que buscan distinciones y equivalencias entre segmentos) pueden concluir con el establecimiento tanto de clases de segmentos equivalentes como de segmentos no equivalentes, distintos, no sustituibles mutuamente. ${ }^{12}$

\footnotetext{
${ }^{11}$ A esta conclusión (o a su contraria) se puede llegar mediante la comparación de las segmentaciones de proferencias que se hallen en alguna de estas situaciones: repetición de una misma proferencia, proferencias diferentes o posiblemente idénticas.

${ }^{12}$ Así, "When these two sets of data are explicitly given, however, it is possible to carry out the rest of the analysis. The fundamental data of descriptive linguistics are therefore the distinctions and equivalences among utterances and parts of utterances" (Harris 1951, p. 33 ).
} 
Una vez que disponemos de los elementos fonológicos, de sus equivalencias y de sus distinciones, es el momento de establecer las relaciones que guardan entre sí esos elementos mediante manipulaciones de tales distinciones sobre la base de su distribución (Cfr. Harris 1951, p. $33)$.

Entre esos procedimientos de manipulación de segmentos está el encaminado a identificar los fonemas; los realizados previamente han logrado identificar una serie de segmentos para la representación de proferencias que, sin embargo, muestran algunos inconvenientes: son muchos y muy restringidos a un reducido número de entornos particulares. Por ello, para identificar un fonema hay que dar los siguientes pasos: en primer lugar hay que establecer la totalidad de los entornos de cada segmento (es decir, la distribución de cada segmento); esto permite, en segundo lugar, indicar para cada segmento el conjunto de segmentos que no comparten con él ningún entorno (es decir, los segmentos con una distribución complementaria a la suya); y, finalmente, en tercer lugar, todo ello hará posible identificar conjuntos de segmentos que no compartan entre sí ningún entorno (es decir, conjunto de segmentos con una mutua distribución complementaria). Pues bien, cada uno de estos conjuntos será concebido como un fonema. "E.g,. segments $[K, k, \mathrm{~K}]^{\text {nota }}$ can all be included in a phoneme $/ k /$. (nota: $K$ indicates back $k$; к indicates front $k$.)" (Harris 1951, p. 61). Por ello, cuando hablamos de la ocurrencia de un fonema en realidad nos referimos a la ocurrencia de alguno de los segmentos que lo integran.

La comprobación de que algunas restricciones en la distribución de los fonemas afectan no a fonemas aislados sino a secuencias de fonemas invita a conceptualizar tales secuencias de fonemas y considerarlos como elementos de interés descriptivo; estos elementos serán los segmentos morfémicos. Una vez establecidos estos, mediante procedimientos que agrupan fonemas y comparan el resultado de su sustitución en las proferencias del corpus:

We now have a list of morphemic segments into which any utterance can be segmented, each of these being uniquely identifiable in terms of phonemic elements, and occurring in stated environments of other morphemic segments (or in stated utterances). An intrinsic part of the definition of each morpheme is the environment for which it is defined. (Harris 1951, p. 171) 
Sobre los segmentos morfémicos se podrán aplicar varias operaciones que permitan establecer entre ellos diferentes relaciones. El propósito es, al igual que en el caso de los fonemas, reducir el número de elementos y las restricciones sobre su ocurrencia. Así "We group mutually complementary morphemic elements into morphemes. This requires that we first list the morphemic elements of our corpus, and note the environments to which each is limited". (Harris 1951, p. 199)

También igual que en el caso de los fonemas, los miembros de un morfema (o clase de elementos morfémicos mutuamente complementarios) se consideran idénticos entre sí.

\section{Representación de proferencias: nociones relacionales básicas}

Los diferentes procedimientos de segmentación y distinción han dado lugar a diversos grupos de elementos descriptivos, tanto fonológicos como morfológicos. Estos elementos pueden ahora usarse para indicar la estructura fonológica y la estructura morfológica de las proferencias.

Para ello, en el nivel fonológico, habría que comenzar por establecer cuáles son las combinaciones posibles de elementos fonológicos, pues no todas las combinaciones ocurren. El modo de hacer esto es agrupando determinados fonemas en clases de fonemas que presentan ciertas semejanzas en común. Por su parte, también los morfemas pueden ser agrupados en clases si se tiene en cuenta que varios de ellos pueden compartir el mismo entorno; es decir, entre ellos se da una relación de sustituibilidad. Una vez precisadas las clases, es posible apreciar que algunas secuencias de éstas son distribucionalmente equivalentes a otras o secuencias de otras clases de morfemas; es decir, ambas ocurren en idénticos entornos en las proferencias y son sustituibles entre sí. Esta equivalencia distribucional expresa una equivalencia en la función sintáctica que cumplen tales secuencias en las proferencias.

We equate any two sequences of classes if one of them is substitutable for the other in all utterances in which either occurs. If the sequence of $A+l y$ is always substitutable for $D$, we write the equation $A l y=D$. This equation means that the range of utterance environments of $A l y$ is identical with that of $D$, or that wherever we find a member of $D$ we may substitute for it not only some other member of $D$ but also some member of $A$ followed by ly. (Harris 1951, p. 263) 
La estructura morfológica de una proferencia se expresa mediante una secuencia de signos (una fórmula o a veces un diagrama) que nombra clases de morfemas. Así, es posible decir que en inglés existen las proferencias con la estructura $N V X$ (nombre-verbo-contorno). Entre una proferencia y su estructura podemos transitar de dos maneras: "we can derive from the formula any utterance of the class which the formula represents [and] given an utterance we can say by what formula it is identified" (Harris 1951, p. 349).

Lo que Harris denomina estructura fonológica o morfológica es lo que en una reconstrucción de la teoría quedaría expresado en las nociones relacionales básicas. Las nociones relacionales básicas de GD serán transcripción fonética, estructura fonológica y estructura morfológica. La definición del conjunto de modelos potenciales de GD deberá reflejar que las tipificaciones de estas nociones proceden de operaciones sobre conjuntos base integrados por elementos fonéticos mínimos y por elementos obtenidos de una elaboración de tales elementos fonéticos mínimos que los agrupa en clases.

Es relevante para establecer las tipificaciones de las nociones de GD atender a cómo se aplica la teoría pero también a las propuestas de Harris cuando señala, aunque no con la suficiente claridad, que se da una especial relación entre una proferencia (o el habla en general) y una de sus descripciones: "a one-one correspondence is maintained between spoken or heard speech and its representation in terms of the elements at any level" (Harris 1951, p. 364). ${ }^{13}$

\section{Relación entre las representaciones de las proferencias: ley fundamental}

Los niveles de descripción (el fonológico y el morfológico) guardan entre sí una estrecha relación, por la cual sus elementos configuran una jerarquía y pueden ser analizados unos en términos de otros: "An element at any of these levels may be defined as consisting of an arrangement of elements of some other level, or as constituting together with other elements of its level some element of another level" (Harris 1951,

\footnotetext{
${ }^{13}$ La poca claridad de esta afirmación procede de la dificultad en saber si aquí Harris utiliza el término 'one-one correspondence' en su sentido matemático (función estricta uno-a-uno y sobre).
} 
p. 364). Pues bien, si entre los elementos de los distintos niveles se establece semejante jerarquización de modo que los elementos de los niveles más altos pueden ser definidos a partir de elementos de niveles más bajos, entonces también entre las descripciones de una misma proferencia obtenidas desde los distintos niveles se establecerá una jerarquización correspondiente de modo que las descripciones ofrecidas desde los niveles más altos podrán ser definidas, obtenidas, a partir de las descripciones ofrecidas desde los niveles más bajos. Esta relación entre las distintas descripciones o estructuras de una proferencia es la que podrá ser expresada como ley fundamental de GD.

En la lingüística estructural (y también en los diferentes desarrollos posteriores) se tenía un especial cuidado en no «confundir los niveles»; es decir, en establecer un ordenamiento entre ellos de modo que la descripción lingüística comenzara por el primer nivel de éste y continuara por los siguientes haciendo uso de la descripción obtenida en el nivel o niveles precedentes. Desde esta perspectiva es muy importante la elección del primer nivel, pues las descripciones realizadas desde ese primer nivel no podrán basarse en ningún otro nivel previo. Aunque algunos importantes lingüistas estructuralistas defendían que el papel de primer nivel debía ocuparlo el nivel fonológico, Harris, como veremos pronto, no opinaba tan rotundamente y, es más, según él, tanto se podía usar la descripción fonológica para obtener la descripción fonológica como a la inversa.

Nuestra propuesta es que la ley fundamental de GD deberá expresar el orden en el que los distintos niveles de la gramática operan y la conexión entre los valores de las nociones asignadas desde esos niveles con la que se compromete el lingüista al aplicar la teoría. Así, si seguimos la propuesta de análisis distribucional de Harris, la ley fundamental de GD vendría a decir que a partir de la transcripción fonética y mediante la aplicación de procedimientos de análisis distribucional se obtendría la estructura fonológica, a partir de la cual, mediante la aplicación de procedimientos de análisis distribucional, se obtendría la estructura morfológica. Los procedimientos de análisis distribucional son, en este caso, procedimientos auxiliares cuya función es obtener la descripción estructural de las proferencias. Esta función es distinta de la que cumplen cuando se emplean para establecer los conjuntos base principales a partir de un corpus representativo. 


\section{Transformaciones}

Acabamos de presentar algunos de los contenidos de GD relevantes para una reconstrucción de esta teoría mediante los recursos de la metateoría estructural. Tales contenidos pertenecen a los ámbitos fonético, fonológico y morfológico. No obstante Harris desarrolla con gran detenimiento otro ámbito de la descripción lingüística, el transformacional, cuya inclusión en una reconstrucción de GD plantea dudas por la incertidumbre existente sobre su pertenencia a la teoría gramatical o al análisis del discurso. En la medida en que el análisis transformacional pertenezca a la teoría gramatical habrá que incluirlo en la reconstrucción de GD y en la medida en que pertenezca al análisis del discurso habrá que excluirlo de la reconstrucción de GD. En este trabajo no tomaremos una decisión al respecto, sino que expondremos los aspectos más relevantes del tratamiento que Harris da a las transformaciones, y lo haremos a modo de prolegómenos para una reconstrucción efectiva futura. Conocer ciertos detalles del tratamiento de Harris servirá, además, para poder identificar sus posibles influencias en el análisis transformacional propuesto por Chomsky en fechas posteriores.

Hemos visto que, según Harris, entre una proferencia y la fórmula que expresa su estructura sintáctica podemos transitar en ambos sentidos. Esto ha sido posible gracias a un proceso que incluye la segmentación del flujo lingüístico que se muestra en las proferencias y el establecimiento de clases de equivalencia cada vez más abarcantes. Estas clases de equivalencia agrupan elementos que son equivalentes en cuanto a su distribución en las proferencias del lenguaje; es decir,

los elementos son equivalentes por tener la misma distribución. Ahora bien, una vez identificados los tipos de oraciones, si pensamos que la emisión de sucesivas y distintas proferencias puede presentar regularidades, podríamos abordar el estudio de la distribución de las oraciones del lenguaje, mostrando así "that sentences of one type are usually followed by others of the same type, or otherwise" (Harris 1951, p. 352). En este caso el ámbito de análisis no sería una proferencia, sino un texto o discurso (o incluso todo el lenguaje) en el que aparecen las proferencias a estudiar.

Harris (1957) aborda la cuestión atendiendo a aquellas oraciones en cuyas estructuras respectivas aparecen repetidas algunas clases de 
morfemas. En algunos casos, entre tales oraciones es posible definir transformaciones que permiten construir (derivar) una de tales oraciones a partir de la otra:

If two, or more constructions (or sequences of constructions) which contain the same $n$ classes (whatever else they may contain) occur with the same $n$ tuples of members of these classes in the same sentence environment (see below), we say that the constructions are transforms of each other, and that each may be derived from any other of them by a particular transformation. For example, the constructions $\mathrm{N} v \mathrm{VN}$ (a sentence) and $\mathrm{N}^{\prime} s$ Ving $\mathrm{N}$ (a noun phrase) are satisfied by the same triples of $\mathrm{N}, \mathrm{V}$ and $\mathrm{N}$ (he, meet, we; foreman, put up, list, etc.); so that any choice of members which we find in the sentence we also find in the noun phrase and vice versa: He meet us, his meeting us; The foreman put the list up, the foreman's putting the list up". (Harris 1957 , p. 147 $)^{14}$

Estas transformaciones pueden entonces ser concebidas como formas variantes de las oraciones, es decir, la aplicación de las transformaciones da lugar a construcciones equivalentes entre sí. ${ }^{15}$

Existen transformaciones reversibles y no reversibles (o unidireccionales), y transformaciones que son reversibles o no en distintos grados o según el contexto, por lo que hay que acompañarlas de ciertas condiciones restrictivas que expresen cuándo lo son o no. Un ejemplo de transformación reversible es la que conecta $\mathrm{N}_{1} v \mathrm{VN}_{2}$ y $\mathrm{N}_{1}^{\prime} s \mathrm{Ving} \mathrm{N}_{2}$, lo cual queda expresado así:

$$
\mathrm{N}_{1} \mathrm{vVN} \mathrm{N}_{2} \leftrightarrow \mathrm{N}_{1}^{\prime} s \text { Ving } \mathrm{N}_{2}
$$

Un ejemplo de transformación no reversible es la que se establece entre oraciones activas y oraciones pasivas, y esto es así porque

every triple of $\mathrm{N}_{1}, \mathrm{~V}$, and $\mathrm{N}_{2}$ in the $\mathrm{N}_{1} \mathrm{vVN} \mathrm{N}_{2}$ 'active' sentence (with some exceptions discussed below) can also be found, in reverse order, in the

14 "Notation: Morpheme and word classes: N (noun) and V (verb) as above; A (adjective) includes large, old, extreme, etc.; $\mathrm{T}$ (article) includes the, a; $\mathrm{P}$ (preposition) includes of, from, to, etc.; C (conjunction) includes and, or, but, etc.; D (adverb) includes very, well, quickly, etc. Classes of affixes (mostly suffixes, some prefixes): na indicates an affix such that $\mathrm{N}+$ na (i.e. the sequence $\mathrm{N}$ na) yields an A word (a word substitutable for A morphemes): papery, cloth-like. Similarly nn after $\mathrm{V}$ yields an $\mathrm{N}$ word: growth; nn after $\mathrm{N}$ yields an $\mathrm{N}$ word: growths, childhood; and so on. And v (tense and verb 'auxiliary' class, \$2.5) includes - ed will, can, etc. S stands for sentence". (Harris 1957, pp. 203-204, nota 2).

${ }^{15}$ Cfr. Harris 1957 , p. 173. 
$\mathrm{N}_{2} \mathrm{v}$ be Ven by $\mathrm{N}_{1}$ 'passive' sentence: The kids broke the window, the window was broken by the kids; The detective will watch the staff, The staff will be watched by the detective. However, some triples satisfy only the second sequences and not the first: The wreck was seen by the seashore (Harris 1957, pp. 147-148). ${ }^{16}$

Por esta razón la transformación pasiva se expresa así: ${ }^{17}$

$$
\mathrm{N}_{1} \mathrm{vVN}_{2} \rightarrow \mathrm{N}_{2} \mathrm{v} \text { be Ven by } \mathrm{N}_{1} \text {. }
$$

Ya hemos indicado que la introducción de las transformaciones por parte de Harris parece motivada por su especial propuesta de análisis del discurso, la cual toma en consideración el estudio de la distribución de oraciones. Dentro de esta perspectiva, una situación en la que las transformaciones pueden ser aplicadas es aquella en la que, por ejemplo, en una secuencia de oraciones (bien por tratarse de oraciones separadas, ${ }^{18}$ bien por tratarse de una oración compleja que incluya dos oraciones $)^{19}$ la ocurrencia de una oración $S_{2}$ está condicionada (positionally bound) a la ocurrencia de una primera oración $S_{1}$. Esta situación, pues, se analizaría considerando a $S_{2}$ una transformación de $S_{1}$.

Harris (1957), aunque no restringe el análisis transformacional a los casos de análisis del discurso, es decir, a los de secuencias de oraciones,

${ }^{16}$ Harris 1957 , pp. 187-188, considera la transformación pasiva con algo más de detenimiento, pero no con el suficiente para que podamos comprender cómo aplicarla y qué otras transformaciones se deben aplicar después para que resulte una proferencia gramatical.

${ }^{17}$ Años atrás, en Harris 1952, encontramos un análisis de la relación entre activa y pasiva diferente: en el ámbito de una propuesta para el análisis del discurso, en la que se requiere el establecimiento de clases de equivalencia entre las oraciones de un lenguaje, se considera que las oraciones pasivas y las oraciones activas pertenecen a la misma clase de equivalencia. La equivalencia entre dos oraciones supone que podemos transformar cualquiera de ellas hasta obtener la otra, de ahí que se hable de transformaciones gramaticales. La equivalencia entre dos oraciones se expresa mediante una igualdad entre las fórmulas que reflejan la estructura de ambos tipos de oraciones. Pues bien, para el caso de las activas y pasivas tendríamos: " $N_{1} V N_{2}=N_{2} V^{*} N_{1}$ where $V$ and $V^{*}$ are respectively active and passive, or passive and active" (Harris 1952, p. 130); es decir, podríamos pasar tanto desde una pasiva a su activa como de una activa a su pasiva. Este doble sentido en el paso de una sentencia a otra es una de las diferencias entre las transformaciones de Harris y Chomsky. Según Tuson (1982, p. 144) "este solo dato es ya indicio de que Harris opera exclusivamente sobre estructuras superficiales".

${ }^{18}$ Como las siguientes: Some groups have rebelled frequently Some only rarely; I've just been over there - Oh, you were there? (cfr. Harris 1957, p. 173).

${ }^{19}$ Como en I met him coming back (cfr. Harris 1957, p. 173). 
son estos los que estudia con detenimiento. ${ }^{20} \mathrm{Un}$ resultado importante de dicho estudio consiste en mostrar cómo "various types of sentences sequence and of complicated sentences are the product of one sentence with the transform of another sentence" (Harris 1957, p. 174). Un ejemplo de tales "is the sequence of assertion and question (John came here \# He did? or Did he? or Who came, did you say?), or question and answer (Who came? \# John or Did John come? \# Yes, he did). In each pair, the composition of one sentence can be described in terms of its predecessor, and is indeed seen to be a transform of it." (Harris 1957, p. 178). Más concretamente, puesto que "each question contains the same words as its neighboring assertion (aside from interchanging you and $I$ ), we can say that it is obtained by a transformation (from that assertion)" (Harris 1957, p. 179). ${ }^{21}$

El estudio de diferentes secuencias de oraciones entre las cuales media una transformación lleva a Harris a distinguir tres grupos en la lista de las principales transformaciones para el inglés: "those that occur in independent sentences $(\mathrm{S} \leftrightarrow \mathrm{S})$; those that occur in sequential sentences $\left(S_{1} \leftrightarrow S_{2}\right)$; and those that occur in sentences that occupy the position of an N-phrase ( $\mathrm{S} \leftrightarrow \mathrm{N}$ )" (Harris 1957, p. 187). La transformación pasiva pertenece al primer grupo, mientras que la transformación que da lugar a las preguntas pertenece al segundo. El tercer grupo es el de las transformaciones que nominalizan una oración, es decir, el de las que la cambian hasta que pueda aparecer en la posición que en otras oraciones ocupan los N-phrases.

Harris señala la existencia de ciertas transformaciones elementales; es decir,

${ }^{20}$ Es decir, analiza transformacionalmente también ciertas oraciones aunque no se incluyan en una secuencia de oraciones constitutivas de un discurso. Un ejemplo de sentencia tal, analizable transformacionalmente pese a no formar parte siempre de una secuencia de oraciones, es Oh, you were there? (cfr. Harris 1957, p. 173).

${ }^{21} \mathrm{El}$ comentario sobre el intercambio de los pronombres you y $I$ indica claramente que para Harris, aquí, las transformaciones sirven para analizar discursos, intercambios de proferencias en situaciones de habla: en una situación de habla los interlocutores utilizan distintos pronombres para referirse a una misma persona (iHas llegado tú hoy? \# Si, yo he llegado hoy) Esta es una cuestión y otra muy distinta es la de cómo relacionar proferencias como é Has llegado tú hoy? y Tú has llegado hoy, que no constituyen un discurso. Esta segunda cuestión es la que afrontará Chomsky, a la que responde afirmando que la relación entre tales proferencias procede de que una primera porción de sus historias derivacionales es idéntica, divergiendo sólo a partir de la aplicación de determinada transformación en el caso de una proferencia pero no en el caso de la otra. 
comparison of various transformations may show that these can be combined out of certain elementary changes, even if these do not occur by themselves. Any transformation which is not obtainable by combining the effect of two or more other simpler transformations will be called an elementary transformation [...] The existence of elementary transformation makes it possible to regard all transformations as compoundings of one or more elementary ones" (Harris 1957, p. 195).

Desde un punto de vista algebraico, "we have here a set of transformations with a base set (the elementary transformations), with products of the base members yielding the various other members of the set" (Harris 1957, p. 196).

Según Harris (1957, p. 92), la mayoría de las transformaciones son "one-one in the sense that for each individual sentence there is one transform and conversely (except for cases of homonymity)." Sin embargo, existen algunas transformaciones many-one, es decir, transformaciones que aplicadas a diferentes oraciones dan el mismo resultado.

Las transformaciones han de cumplir ciertas condiciones de aplicación; una transformación no puede aplicarse siempre a cualquier oración, es decir, las transformaciones "are restricted to particular structural environments." Además, no puede estar compuesta por cualquier transformacion elemental aplicada en un orden cualquiera. ${ }^{22}$

Hay entre las oraciones de un lenguaje las procedentes de la aplicación de alguna transformación y las que no; estas últimas son las oraciones elementales $\mathrm{u}$ oraciones nucleares (kernel sentences). Cada oración no nuclear se obtendrá de una o varias oraciones nucleares mediante una o varias transformaciones. La fórmula o construcción que expresa la estructura de una oración nuclear la podemos llamar construcción nuclear.

kernels generally contain very few constructions; and applying transformations to these few constructions suffices to yield all the many sentence constructions of the language. The kernel constructions of English seem to be only these:

$\mathrm{N}$ v V (for V that occur without objects);

$\mathrm{N} v \mathrm{~V} P \mathrm{~N}$ (for P N that have restricted co-occurrence

22 "The successive application of elementary transformations can be called their product" (Harris 1957, pp. 195-196). 
with particular V);

$$
\begin{aligned}
& \mathrm{N} \text { v V N; } \\
& \mathrm{N} \text { is } \mathrm{N} ; \\
& \mathrm{N} \text { is } \mathrm{A} ; \\
& \mathrm{N} \text { is } \mathrm{P} \mathrm{N} ; \\
& \mathrm{N} \text { is } \mathrm{D} .
\end{aligned}
$$

In addition there are a few minor constructions" (Harris 1957, p. 198).

Si las transformaciones pueden dar lugar a una ilimitada variedad y longitud de oraciones es debido a "the unbounded repeatibility of various sequential transformations" (Harris 1957, p. 199). Además de éste, otro de los efectos de las transformaciones en la estructura del lenguaje es que pueden dar cuenta de ciertas similaridades entre oraciones, considerando a éstas a partir de las mismas oraciones nucleares pero mediante diferentes transformaciones. ${ }^{23}$

Harris considera que existen aplicaciones de las transformaciones en algunos campos del ámbito de la lingüística que las hace especialmente útiles. La más importante de estas aplicaciones tiene que ver con sus peculiaridades semánticas. Así, pese a que el significado no puede ser estudiado directamente en el seno de la lingüística estructural, según Harris, sí sería posible establecer procedimientos de evaluación práctica del significado mediante las transformaciones. Y esto debido a que "many sentences which are transforms of each other have more or less the same meaning, except for different external grammatical status" (Harris 1957, p. 202). ${ }^{24}$ El significado al que se refiere Harris es lo que llama 'contenido de información' [information content ${ }^{25}$ De esta

\footnotetext{
${ }^{23}$ Los ejemplos serían las oraciones: Mary has a sad fate, Mary's fate is sad, Mary's fate is a sad one, Mary's is a sad fate; estas se obtendrían mediante diferentes transformaciones a partir de alguna de las oraciones nucleares siguientes: Mary has a fate, Fate is a fate, Fate is sad (Cfr. Harris 1957, pp. 199-200).

24 "This is not surprising, since meaning correlates closely with range of occurrence, and transformations maintain the same occurrence range" (Harris 1957, p. 202). La posición de Harris acerca de la supuesta inocuidad de las transformaciones sobre el significado no parece muy firme si atendemos a la siguiente cita: "In many cases the same $S_{1} S_{2}$ sequence may occur with various connectives between them, or with various transformations on $S_{2}$, with the resultant meaning either virtually the same or different according to the connectives and the transformations in question" (Harris 1957, p. 186).

${ }^{25}$ Cfr. Harris 1957, p. 150.
} 
manera las transformaciones se muestran como "a posible tool for reducing the complexity of sentences under semantically controlled conditions" (Harris 1957, p. 203). La disminución de la complejidad es también un logro obtenible en el análisis de discursos y de secuencias de oraciones realizados identificando las oraciones nucleares implicadas y las transformaciones que operan a cada caso.

Como hemos podido apreciar, Harris, en la segunda mitad de los 50 , se preocupó de señalar algunos de los rasgos de aquellas gramáticas que incorporan transformaciones frente a las gramáticas propias de la tradición estructural o descriptiva: "Those constructional features of grammar which are well known from descriptive linguistics are in general limited to the kernel. In the kernel, the constructions are built up a concatenation of various included constructions, down to morpheme classes" (Harris 1957, p. 200). "All sentences which are described in constructional terms must have a specific constituent analysis, since the constructional analysis proceeds in terms of constituent analysis" (Harris 1957, p. 201). A diferencia de lo que se lleva a cabo en la lingüística estructural, las transformaciones establecen una relación entre oraciones completas, modificándolas de modo que el análisis en constituyentes inmediatos desaparece: "Some of the cruces in descriptive linguistics have been due to the search for a constituent analysis in sentence types where this does not exist because the sentences are transformationally derived from others. For this and other reasons a language cannot be fully described in purely constructional terms, without the transform relation" (Harris 1957, p. 201).

Es obvio que esta comparación entre el análisis distribucional tradicional y sus propias propuestas es realizada por Harris desde el punto de vista de un análisis transformacional no de un discurso (o secuencia de oraciones) sino de un análisis transformacional de oraciones independientes (discursivamente hablando). Es decir, en estos años, la propuesta de Harris cabe considerarla como integrante de una teoría gramatical y no del análisis del discurso, y por tanto deberíamos concluir que está en consonancia con la propuesta que Chomsky estaba realizando en ese mismo periodo, es decir la propuesta hecha en GCH.

El estudio de los lenguajes naturales mediante reglas transformacionales es algo que se asocia inmediatamente con la obra de Chomsky. Sin embargo hemos visto cómo Harris introduce y desarrolla el tema 
de un modo no incidental. Los historiadores y filósofos de la lingüística no coinciden al enjuiciar la influencia que la obra de Harris tuvo en el tratamiento y uso de las transformaciones en la corriente generativa transformacional. Las opiniones van desde los que piensan que tal influencia no existió, puesto que el asunto que Harris tenía en mente era otro, era el análisis del discurso; ${ }^{26}$ pasando por quienes consideran que tal influencia existió a pesar de que a Harris le interesara el análisis del discurso; ${ }^{27}$ y van hasta los que consideran que la teoría transformacional inicial de Chomsky no se diferencia de la teoría transformacional de Harris salvo en ciertos ingredientes metateóricos. ${ }^{28}$

Más allá de decidir a quién corresponde el mérito de haber incluido las transformaciones en las gramáticas de los años 50, si a Harris o a Chomsky, lo fundamental es poder reconstruir los distintos usos que se le dieron a las transformaciones, pues esa reconstrucción ayudará a comprender los objetivos que guiaban la elaboración de gramáticas en esos años, los cambios producidos a partir de entonces y la naturaleza y alcance del conocimiento que brinda la lingüística como ciencia.

\section{Referencias}

Chomsky, N., 1975 [1955], The Logical Structure of Linguistic Theory, Plenum, Nueva York.

Díez, J. A. y P. Lorenzano (eds.), 2002, Desarrollos actuales de la metateoría estructuralista: problemas y discusiones, Universidad Nacional de Quilmes, Buenos Aires.

\footnotetext{
${ }^{26}$ En la obra de Chomsky, las reglas transformacionales "se inspiraban, y eran esencialmente reinterpretaciones suyas, en las reglas que se denominaban de igual forma propuestas por su maestro Zellig Harris en su intento de aplicar los métodos de la lingüística estructural al análisis del discurso" (Newmeyer 1980, p. 44).

${ }^{27}$ Ruwet (1967, pp. 301-319) es un buen ejemplo. Según este autor, una de las aportaciones más importantes de Harris "reside en los análisis concretos que ha dado de las transformaciones en inglés, análisis cuyos resultados han sido incorporados, en gran parte, en forma modificada a veces, a la gramática generativa del inglés. Es así como en el análisis del auxiliar, la interpretación de do como soporte fonemático de un afijo no afijado, el análisis de los pronombres relativo-interrogativos, el de los pro-sintagmas, las grandes líneas del análisis de la nominalizaciones, etc., se encuentran ya en (Harris 1957, passim y, especialmente, pp. 195-202)” (Ruwet 1967, p. 311).

28 "Chomsky's transformational theory is Harris's, with three important differences: first, that Chomsky's is conceptualiscally interpreted as a theory about psychological states, second, that Chomsky's is constructed as a theory of generative grammar, and third, that Chomsky's is presented as a scientific theory. Thus, Chomsky's early transformationalism is Harris's transformationalism without nominalism and the ascientific outlook idiosyncratic to Harris's own approach to natural languages" (Katz 1981, p. 30).
} 
Estany, A. y D. Quesada (comps.), 1997, Actas del II Congreso de la Sociedad de Lógica, Metodología y Filosofía de la Ciencia en España, Servei de Publicacions de la Universitat Autónoma de Barcelona, Barcelona.

Falguera, J. L., A.J.T. Zilhao, C. Martínez y J.M. Sagüillo (comps.), 2003, Palabras y pensamientos: una mirada analítica, Universidad de Santiago de Compostela, Santiago de Compostela.

Harris, Z.S., 1981, Papers on Syntax, Reidel, Dordrecht.

—, 1957, "Co-occurrence and Transformation in Linguistic Structure", Language, vol. 3, no. 33, pp. 283-340. (Citado por su reedición en Harris 1981, pp. 143-210).

—, 1954, "Distributional Structure", Word, vols. 2-3, no. 10, pp. 146-162. (Citado por su reedición en Harris 1981, pp. 3-22).

—, 1952, "Discourse Analysis", Language, vol. 1, no. 28, pp. 1-30. (Citado por su reedición en Harris 1981, pp. 107-142).

—, 1951, Structural Linguistics, University of Chicago Press, Chicago.

Hymes, D. y J. Fought, 1981, American Structuralism, Moutons, La Haya.

Katz, J.J., 1981, Language and Other Abstract Objects, Basil Blackwell, Oxford.

Marcus, S., 1967, Algebraic Linguistics; Analytical Models, Academic Press, Nueva York.

Mcquown, N. A., 1952, "Review of Methods in Structural Linguistics, by Z.S. Harris", Language, no. 28, pp. 495-504.

Newmeyer, F.J., 1980, Linguistic Theory in America - The First Quarter-Century of Transformational Grammar, Academic Press, Nueva York.

Peris-Viñé, L.M. (comp.), 2012a, Filosofía de la ciencia en Iberoamérica: metateoría estructural, Editorial Tecnos, Madrid.

— 2012b, "Estructura parcial de la gramática estándar del castellano", en Peris-Viñe 2012a, pp. 225-258.

—, 2011, "Actual Models of the Chomsky Grammar", Metatheoria, vol.1, no. 2, pp. 195-225.

—, 2003, "Concepciones ontológicas en Bloomfield y en Chomsky", en Falguera, Zilhao, Martínez y Sagüillo 2003, pp. 239-270.

—, 2002, "Relaciones interteóricas en la lingüística actual", en Díez y Lorenzano 2002, pp. 333-362.

—, 1997, "Los lingüistas naturalistas contra sus teorías", en Estany y Quesada 1997, pp. 44-47.

—, 1996, "Caracterización de las nociones básicas de la Gramática de Chomsky", Ágora, vol. 2, no. 15, pp. 105-124.

Plötz, S. ed., 1972, Transformational Analysis: The Transformational Theory of Zellig Harris and its Development, Athenäum Verlag, Francfort del Meno.

Ruwet, N., 1967, Introduction a la grammaire générative, Librairie Plon, París. Serrano, S., 1975, Elementos de lingüística matemática, Anagrama, Barcelona.

Tuson, J., 1982, Aproximación a la Historia de la Lingüística, Teide, Barcelona. 
Recibido el 8 de septiembre de 2011 Aceptado el 30 de noviembre de 2011 